\title{
Modélisation des équilibres liquide/vapeur du système $\mathrm{H}_{2} \mathrm{O}-\mathrm{H}_{2} \mathrm{NCH}_{2} \mathrm{CH}_{2} \mathrm{NH}_{2}$ (EDA)
}

\author{
R. Tenu, C. Duriche, H. Delalu et J.J. Counioux \\ CNRS, Université Claude Bernard Lyon I, Groupe SNPE - UMR « Hydrazines et \\ Procédés », 43 Bd. du 11 novembre 1918, 69622 Villeurbanne Cedex, France
}

\begin{abstract}
The liquid-vapour equilibria of the binary system water-ethylenediamine have been experimentally studied under isobaric conditions on the one hand at 100,400 and 700 Torr, and on the other hand at 2,69 and 3,65 atm.

The phase equilibrium modelling, as a function of temperature and pressure, has been carried out by means of the quasiideal model, according to both two fundamental assumptions : the basicity of EDA and the solvation of chemical species that constitute the solution. The thermodynamic analysis of experimental data leads mainly to the following results :

- the hydrolysis enthalpy of EDA reaches around $50 \mathrm{~kJ}$,

- the global degree of solvation linearly varies with the logarithm of pressure,

- the azeotropic phenomenon disappears above $180 \mathrm{kPa}$,

- within the range of pressure studied, experimental data and calculation results are in very good agreement.
\end{abstract}

\section{INTRODUCTION}

Les opérations de distillation sont couramment utilisées pour extraire des produits purs de solutions de synthèse complexes. L'existence de phénomènes d'azéotropie ou encore la formation de " saddle points » limitent souvent les possibilités de séparation. L'homme de l'art s'emploie alors à « casser » l'azéotrope et fait souvent appel aux techniques de relargage. Une alternative, particulièrement judicieuse dans le cas des unités de production en continu, consiste à jouer sur un autre paramètre en mettant en ouvre des distillations en surpression ou sous pression réduite. Malheureusement, la définition et la mise au point de tels procédés impliquent la connaissance d'un ensemble de données expérimentales qui font pratiquement toujours défaut pour une application particulière.

La modélisation des phénomènes constitue alors une alternative avantageuse puisqu'elle permet, d'une part, la sélection des adjuvants et, d'autre part, une optimisation des conditions d'extraction. Le modèle quasiidéal, mis au point au laboratoire depuis une quinzaine d'années, a été appliqué ici au calcul prédictif des équilibres liquide/vapeur du système Eau-Ethylène Diamine (ou EDA de formule $\mathrm{H}_{2} \mathrm{NCH}_{2} \mathrm{CH}_{2} \mathrm{NH}_{2}$ ).

\section{LE MODELE QUASIIDEAL}

Par hypothèse la solution quasiidéale est constituée d'entités physicochimiques dont les activités sont égales à leur probabilité d'existence. Cette extension du concept d'idéalité permet de conserver le formalisme simple des équations d'équilibre entre deux phases idéales $\alpha$ et $\beta$ :

$$
R \sum_{i} x_{i}^{*} d\left[\ln \left(\frac{x_{i}^{\beta}}{x_{i}^{\alpha}}\right)\right]+\Delta h_{\alpha \beta} d\left(\frac{1}{T}\right)+\frac{\Delta v_{\alpha \beta}}{T} d P=0
$$




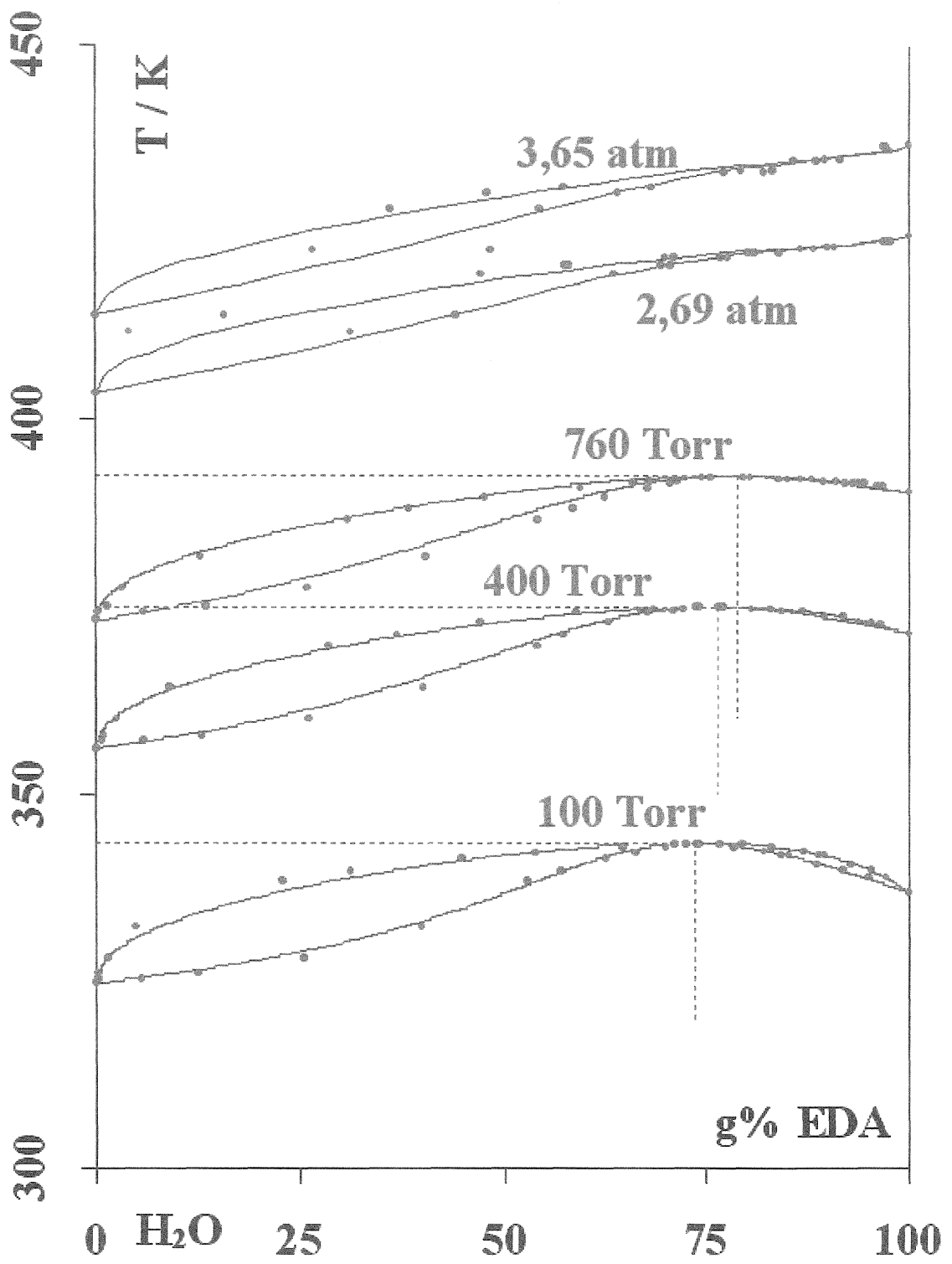

où $\mathrm{x}_{\mathrm{i}}^{*}$ et $\mathrm{x}_{\mathrm{i}}^{\varphi}$ sont respectivement les fractions molaires des entités $\mathrm{i}$ dans l'état de référence choisi (composition, température $T_{\alpha \beta}$, pression $P_{\alpha \beta}$ ) et dans la phase $\varphi$. Les grandeurs $\Delta \mathrm{h}_{\alpha \beta}$ et $\Delta \mathrm{v}_{\alpha \beta}$ représentent respectivement les variations d'enthalpie et de volume d'une mole de $\alpha$ en $\beta$ à la température $T$. 


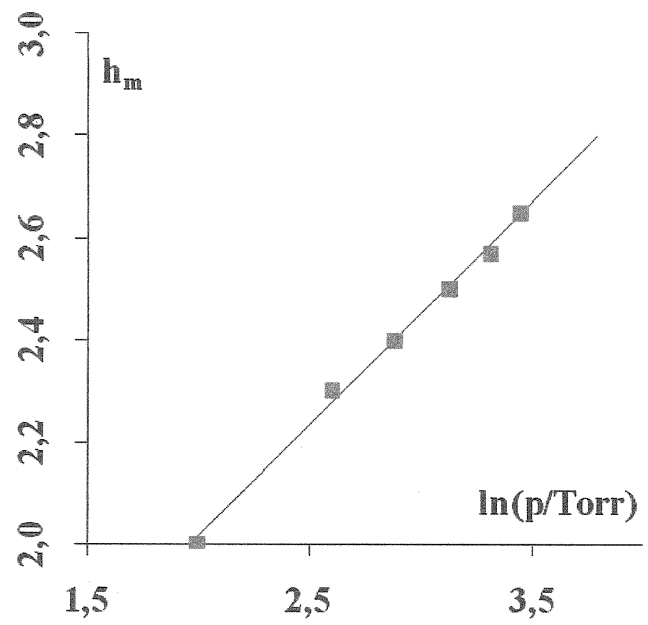

Figure 2 : Evolution de $h_{m}$ en fonction du logarithme de la pression

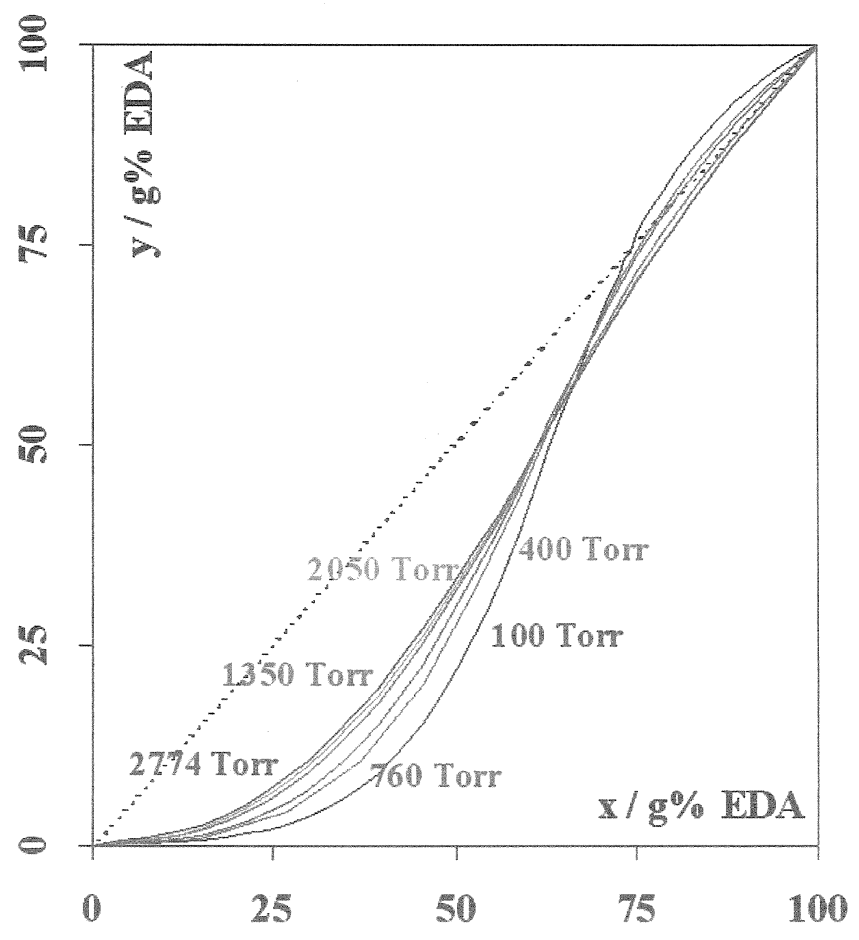

Figure 3 : Courbes $y_{\text {vapeur }}=f\left[x_{\text {liquide }}\right]$ en fonction de la pression 
A température constante ou sous pression imposée, cette équation se simplifie et son intégration est relativement aisée (équations 2 et 3 respectivement) :

$$
\begin{aligned}
& R \sum_{i} x_{i}^{*} \ln \left(\frac{x_{i}^{\beta}}{x_{i}^{\alpha}}\right)+\int_{T_{\alpha \beta}}^{T} \Delta h_{\alpha \beta} d\left(\frac{1}{T}\right)=0 \\
& R \sum_{i} x_{i}^{*} \ln \left(\frac{x_{i}^{\beta}}{x_{i}^{\alpha}}\right)+\int_{P_{\alpha \beta}}^{P} \frac{\Delta v_{\alpha \beta}}{T} d P=0
\end{aligned}
$$

\section{MODELISATION DES EQUILIBRES LIQUIDE/VAPEUR}

Les équilibres liquide/vapeur du système $\mathrm{H}_{2} \mathrm{O}$-EDA ont été étudiés expérimentalement sous 100,400 et 760 Torr [1] puis sous 2,69 et 3,65 atm [2]. Le diagramme de phases se caractérise par l'existence d'un azéotrope qui disparaît sous pression. Des essais exploratoires systématiques à l'aide du modèle quasiidéal ont montré que les seules interactions fortes à l'état liquide découlent du caractère basique des molécules d'EDA et de la solvatation des différents constituants de la solution. Ces phénomènes ont été pris en compte sous la forme d'un degré de solvatation moléculaire $h_{m}$ selon l'équilibre réactionnel suivant :

$$
\begin{aligned}
((\mathrm{EDA}))+\mathrm{h}_{\mathrm{m}}\left(\left(\mathrm{H}_{2} \mathrm{O}\right)\right) & \leftrightarrows\left(\left(\mathrm{EDAH}^{+} . \mathrm{aq}\right)\right)+\left(\left(\mathrm{OH}^{-} . \mathrm{aq}\right)\right) \\
\Delta \mathrm{H}_{\mathrm{eq}} & \approx 50{\mathrm{~kJ} . \mathrm{mol}^{-1}}^{-1}
\end{aligned}
$$

Dans ces conditions, l'exploitation des données expérimentales a permis de :

- calculer une enthalpie de réaction voisine de $50 \mathrm{~kJ}$,

- déterminer l'évolution du degré de solvatation moyen $h_{m}$ en fonction de la pression. Comme le montre la figure 2 , le paramètre varie de façon pratiquement linéaire avec le logarithme de la pression imposée dans le domaine expérimental considéré,

- mettre en évidence une pression limite, de $180 \mathrm{kPa}$, au-dessus de laquelle le phénomène d'azéotropie n'est plus observé.

La figure 3 représente, sous différentes pressions, les courbes de composition y de la phase vapeur en fonction de la composition $\mathrm{x}$ de la phase liquide en équilibre. On peut remarquer le déplacement de la composition du point azéotropique jusqu'au point $\mathrm{x}=\mathrm{y}=1$ à 1350 Torr.

La figure 1 met en évidence la pertinence du modèle. Une parfaite corrélation est observée entre les résultats du calcul et ceux de l'expérience sous les cinq pressions étudiées. Ces résultats autorisent le calcul prédictif de tout diagramme isobare des équilibres liquide/vapeur jusqu'à au moins $0,5 \mathrm{MPa}$.

\section{Références}

1. J. Schmelzer et K. Quitzsch, Z. Phys. Ch., 252, 5/6, p.280-288 (1973)

2. M. Hirata, S. Suda, T. Hakuta et K. Nagahama, J. Ch. Eng. (Japan)., 2, 2, p.143-149 (1969) 